\title{
Multi-Path Routing Protocol for Vehicular Ad-hoc Networks
}

\author{
H. Ahmed \\ Ain Shams University \\ Cairo, Egypt
}

\author{
Shouman E. I. Shouman \\ Modern Academy for Engineering and Technology \\ Maady, Cairo \\ Egypt
}

\begin{abstract}
In this paper, we explore the impact of the vehicular density on routing protocol design for vehicular ad hoc networks (VANET). Specifically, we focus on multi-path-based routing protocols designed for vehicular communications in urban areas. We find that the vehicular density greatly impacts the routing in urban areas. However, low vehicular density scenarios have not been well treated in the existing routing protocols. In this paper, we propose a new routing protocol called multi-path routing (MPR) for VANETs. MPR is a beacon less routing protocol with carry-andforward mechanisms that forwards multiple copies from the packet and eliminates unneeded copies at the intersections. The proposed protocol creates enough diversity to reach the destination vehicle with shorter end-to-end delay. The paper shows via simulation that the proposed multi-path protocol is superior to other proposed protocols, especially in low vehicular density scenarios. The results show that MPR achieves shorter end-to-end delay and higher delivery ratio compared with the existing routing protocols for urban VANET communications.
\end{abstract}

\section{General Terms}

Vehicular ad-hoc networks, Routing

\section{Keywords}

End-to-end delay,routing, VANET

\section{INTRODUCTION}

Vehicular ad hoc networks (VANET) technology enables communication between vehicles, or vehicles and road-side units (RSU) through a wireless communication devices installed on the vehicles. One of the most important goals of VANET is providing safety applications for passengers. Moreover, VANET also provides comfort applications to the users, for example; mobile e-commerce, weather information, Internet access, and other many multimedia applications. Routing is a fundamental operation for vehicular communications to select a source-to-destination path in a VANET. The important goal of unicast routing protocols in VANET communications is to transmit data from a source to a destination via multi-hop path. Some VANETs applications have end-to-end delay constraints than high data rates, such as accident avoidance ap- plications. Therefor, end-to-end delay is very important issues in VANET routing design.

VANETs connectivity often changes, especially when the vehicular density is low, this usually leads to disconnected networks. The low traffic density can create partitions in the network and packets may either get dropped or face higher delays [8]. In this situation where direct end-to-end path between source and destination can be considered as nonexistent, a regular ad hoc routing protocol with complete path discovery mechanism is not feasible since the routing path is usually disconnected due to the intermittent nature of network links. To overcome this problem, vehicles can be used as carriers to deliver messages via carry-and-forward whenever forwarding option via wireless transmission is not available.

Many papers proposed for VANET routing in urban scenarios. Most of routing protocols in urban areas are position based routing that depend on greedy perimeter stateless routing (GPSR). GPSR [5] protocol uses greedy forwarding to forward the packet from a source to a destination. In greedy forwarding, GPSR tries to bring packets closer to the destination in each hop by using geographic information. However, in many cases, greedy forwarding can lead to areas where there is no neighbour closer to the destination vehicle except for the current forwarding vehicle.

Greedy traffic-aware routing (GYTAR) [4] protocol uses digital maps to identify the position of intersections and location service to get the destination location. It tries to select a forward path with higher vehicular density and shorter distance. The protocol consists of two parts, namely dynamic junction selection procedure and forwarding strategy between two involved intersections. For each intersection, the protocol calculates a score for each candidate road segment that depends on the vehicular density and the Euclidean distance for the destination from this road segment. The candidate intersection with the highest score will be selected. After the next intersection is selected, the protocol uses greedy forwarding to forward the packets toward the selected intersection. Moreover, GYTAR uses carry-and-forward as one of its routing strategies to face the network disconnection. Enhancement on GYTAR proposed on [1] to select the global optimum path.

Backbone assisted hop greedy routing (BHAG) |6] selects the forwarding path with the minimum number of intersections. This is because the shortest path, or the path with the highest connectivity, may include numerous intermediate intersections. As a result, this will yield to a routing paths with a higher hop count. Moreover, it scores the connectivity of the streets based 
on the number of lanes. On the other side, street-centric routing protocol based on micro topology (BHAG) [2], represents the city on a transfer graph, where each edge represents micro topology while the vertex represents an intersection. Micro topology consists of vehicles and wireless links among vehicles along a street. The weight for edges depend the on vehicles mobility, signal fading, wireless channel contention, and existing data traffic. Multiple path for video streaming proposed in [7], it distributes the traffic into a set of two or three paths for load balancing.

All of the previous mentioned routing protocols depend on sending a single path of the packet and try to select the path with the highest vehicular to avoid network disconnection. However, this single path may face red traffic light or disconnected segment in low vehicular density scenarios. Therefore, most of previous protocols propose the simulation result under high traffic density or in a small area. For example, BHAG protocol obtains their results with 600 nodes in an area of $3 \mathrm{~km} \times 3 \mathrm{~km}$. On the other hand, BHAG started the simulation with 100 to 300 nodes; however, in an area of $2 \mathrm{~km} \mathrm{x}$ $1.5 \mathrm{~km}$. Moreover, GYTAR started the simulation with 100 to 350 nodes in area of $2.5 \mathrm{~km} \times 2 \mathrm{~km}$. Nevertheless, one of VANET mobility characteristic is that it has low vehicular density as well as high Vehicular density. Therefor, we need to consider low vehicular density scenarios in the simulation. Scenarios with low vehicular density have higher probability of network disconnection [9]. As a result the packets will suffer from long end-to-end delay due to queuing in the buffer and this will increase the packet loss probability caused by timeout or overflow in the queue [8].

This paper focus on developing multi-path intersection based routing (MPR). It is a beacon less routing protocol with carry-andforward mechanism. The proposed protocol deals with low vehicular density by forwarding multiple copies of the packets to different directions. Our approach is to replicate data packets and distribute them to different road segments and eliminates the unneeded copies at the intersections to minimize the overhead. The protocol increases the chance of reaching the destination with short endto-end delay. Moreover, on the straight road segment where there are no alternative paths, the protocol greedily forwards packets to the next intersection that leads towards the destination. The main contributions of this paper are as follows: 1) It proposes a new protocol for VANET in urban that can deal efficiently with low vehicular density scenarios. 2) It analyze the proposed protocol at low density scenarios and their impact on the routing performance. The rest of this paper is organized as follows. Section II introduces the system model and MPR protocol design. Section III proposes the performance evaluation in terms of packet delivery rate and average end-to-end delay. Finally, Section IV presents the conclusions.

\section{PROPOSED ROUTING PROTOCOL}

\subsection{System Model}

In our MPR design, we assume that each vehicle has the capability to obtain the road map data and its position information, which we consider as a valid assumption since nowadays most of the vehicles have a GPS device. In addition, it is assumed that the source vehicle acquires destination's position via a location service, which is beyond the scope of our design and will be not discussed in this section. Once the destination vehicle's location is obtained, the information is carried in the packet. Therefore, the intermediate nodes do not to use the location service. However, due to the dynamic nature of a vehicular network, the destination vehicle may have already left the area by the time packets arrive at the initial loca- tion. In this case, the packet carrier will obtain the new location of the destination vehicle via location service and forward the packets toward the new location. Further, we presume the use of location service is limited only to acquiring the destination node location. Also, it is assumed a grid model for city environment. This model is based on Manhattan grid mobility model, also known as city section mobility model.

\subsection{MPR Modes}

MPR routing has three modes that depend on the location of the forwarding vehicle - the vehicle that has a packet and wants to forward it - and the vehicular density status. Depending on the location of the forwarding vehicle, MPR can switch from greedy forwarding mode to multi-path forwarding mode. This switching happens when the node moves from a road segment to an intersection. A road segment is a segment between two adjacent intersections. On the other hand, MPR switches from the greedy forwarding mode to the carry mode if the forwarding vehicle does not find next hop for the packet. Actually, this mode mostly exists at low vehicular density scenarios. We will describe each mode in detail as following.

The first mode is greedy forwarding mode. In this mode, The current location of the forwarding vehicle and the destination are stored in the packet header to enable the neighbours to calculate their progress toward the destination and their distance to the forwarding vehicle. The only available information for each vehicle is its own location from the GPS, the previous forwarding vehicle location, and the destination location, extracted from the packet header. Each neighbour vehicle estimates how it is suited as a next hop for this packet. This estimation depends on two parameters. The first parameter is the distance between the neighbour and the forwarding vehicle, while the second parameter is the distance between the neighbour and the destination. All neighbours that receive the packet check if they are closer to the destination than the forwarding vehicle. If this condition is true, the neighbour vehicle will start a timer that depends on the distance between this node and the forwarding node:

$$
\text { Delay }=\frac{D-R}{R}
$$

where $\mathrm{R}$ is the communication range and $\mathrm{D}$ is the Euclidean distance from the neighbour vehicle to the forwarding vehicle as shown in greedy forwarding algorithm . Therefore, a neighbour vehicle with longer distance will start with forwarding this packet first and will be the next hop. Consequently, achieve more progress toward the destination, this will decrease number of hops and decrease end-to-end delay. When one vehicle forwards the packet, all other nodes in this area will drop their packet and stop forwarding. The MPR algorithm shows this mode in detail at check forwarding timer procedure. Simultaneously, the forwarding vehicle will start to overhear if one neighbour send the packet or not and keep a copy of this packet at their carry buffer. Carry buffer, is a buffer contains the packets that already have been send by this node. If the forwarding vehicle overhears one neighbour forwarded this packet, it will drop this packet from the carry buffer. Otherwise, after the overhearing timer out without overhearing any one from the neighbour forwarded the packet, the forwarding vehicle will switch this packet to carry mode which is the second mode.The MPR algorithm shows this process in detail at start overhearing timer algorithm. 


\subsection{Pseudo code for MPR}

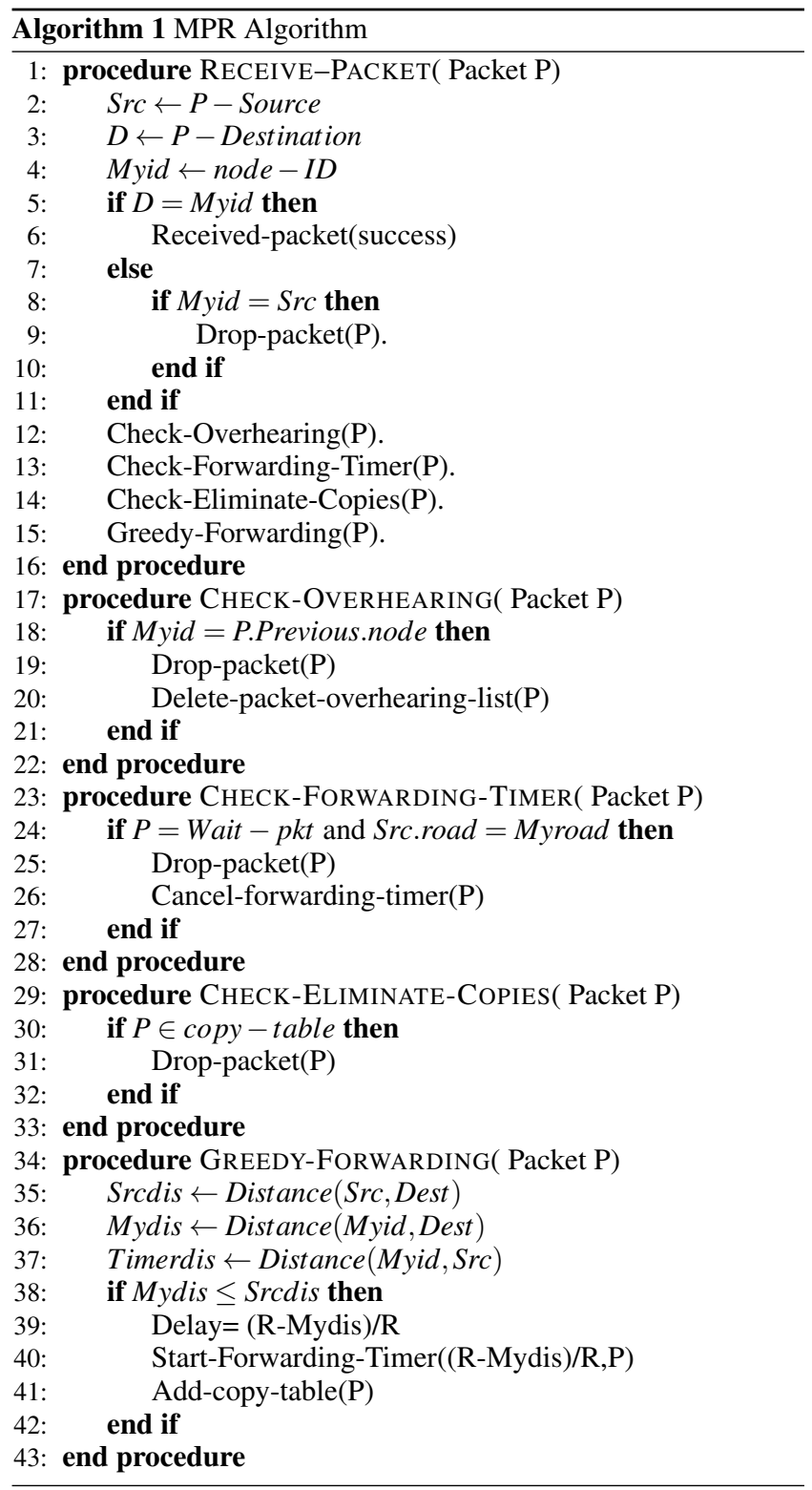

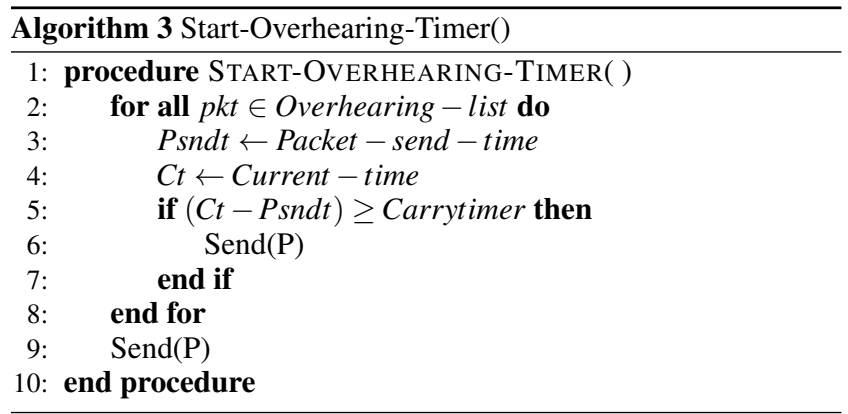

MPR switches to carry mode when a forwarding node gets stuck in to a forwarding area with no neighbours, this happens mostly in low vehicular scenarios. A recovery strategy must be started; otherwise the packet will be dropped. This strategy is timer based. The forwarding vehicle will add this packet to carry buffer. This buffer contains all packets that do not have next hop. Depending on a timer, each period, the forwarding vehicle will rebroadcast the packet and start overhearing. As a result, if one neighbour exists in the forwarding area and closer to the destination than the forwarding vehicle, this neighbour will rebroadcast the packet and the forwarding vehicle will dropped it after overhearing happen. The MPR algorithm shows this process in detail at check overhearing procedure

Finally, the third mode is intersection mode. At the intersection area that defined by the digital map, MPR operates under intersection mode. At the intersections, MPR may forward the packet or drop it. MPR forwards and eliminates the multiple copies of the packet at the intersections. The packet that will reach the intersection later will be discarded. MPR will forward the packet if it did not reach this intersection before. Therefore, each vehicle will check if it had received this packet or not before starting forwarding process. If the vehicle forwarded this packet before, the packet will be dropped. Otherwise, the node will broadcast the packet and start the overhearing timer. After that, each neighbour vehicle in this intersection will check if it located in a candidate road segment or not by using the digital map and the destination location in the packet header. If this is true, it will start the forwarding timer as mentioned before. In this mode, the vehicle drops the packet and stops the forwarding timer if one neighbour in the same road segment forwarded the same packet. Before dropping the packet, the vehicle must make sure that it is located in the same road segment of the forwarding neighbour. This condition because the node may overhear one node forwarded the packet but in another road segment and MPR may forward the same packet in more than one road segment. Finally, the forwarding node will drop the packet from overhearing list after it make sure that there is one neighbour forwarded the packet.

\section{SIMULATION RESULTS}

This section presents the performance evaluation of MPR to investigate the performance impact of multi-path on routing protocols. We implement our proposed MPR protocol in NS-2 (V-2.34). For comparison, we implement GPSR and BHAG explained in the Introduction Section. We make two modifications on GPSR to be more suited for VANET and for fair comparison with MPR. The first modification is the addition of the location service on GPSR to get the location of the destination vehicle, while the second modification is the addition of the carry-and-forward strategy. 
The simulation scenarios are configured in a $3 \mathrm{~km} \times 3 \mathrm{~km}$ urban grid model with different vehicular densities ranging from 5 vehicles $/ \mathrm{km}$ to 30 vehicles $/ \mathrm{km}$. We use VanetMobiSim [3] to generate realistic vehicle mobility. Table 1 summarizes the configuration parameters used in the simulation.

Table 1.

Parameters

\begin{tabular}{|l|l|}
\hline \multicolumn{2}{|c|}{ Parameters } \\
\hline Simulation Parameter & Value \\
\hline Area & $3 \mathrm{~km} \times 3 \mathrm{~km}$ \\
\hline Vehicular density (vehicles/km) & $5,10,15,20,25,30$ \\
\hline Speed (m/sec) & 5 to 15 \\
\hline Simulation time (seconds) & 600 \\
\hline Traffic model & CBR Traffic \\
\hline CBR rate (packets/second) & 2 \\
\hline Transmission range (m) & 250 \\
\hline Channel date rate (Mbps) & 2 \\
\hline Packet size (bytes) & 256 \\
\hline Number of sessions & 1 \\
\hline Number of intersections & 16 \\
\hline
\end{tabular}

Four main important performance metrics are considered. The first metric is the end-to-end delay defined as the difference between the time a data packet arrives at its destination and the time the same packet is originated by the source. This time includes all possible delays as follows

$$
\text { Delay }=\text { Queuedelay }+ \text { Carrydelay }+ \text { Propdelay }+ \text { Trdelay },
$$

where Queuedelay is the queuing delay, Carrydelay is the Carry Mode delay, Propdelay is the propagation delay over the wireless channel, and Trdelay is transmission delay. The second metric is the packet delivery ratio (PDR) defined as the ratio of the total number of the data packets received by the destination to the the total number of the data packets sent by the traffic sources. Finally, the third metric is the routing overhead defined as follows

$$
(\text { Overhead }) \text { packets }=\frac{\text { Number of transmitted packets }}{\text { Number of received data packets }}
$$

However, the routing overhead in MPR represents data and the alarm packets, while the overhead in modified-GPSR and BHAG represents beacon and data packets. In addition, the beacon and alarm packets are much smaller than the data packets. Therefore, for fair comparison with MPR, we consider the fourth metric that represents the routing overhead in the number of transmitted bits as in [2] . It is defined as follows

$$
\text { (Overhead) bits }=\frac{\text { Number of transmitted bits }}{\text { Number of received data bits }} .
$$

Fig. 1 shows the average end-to-end delay against the vehicular density for MPR, BHAG, and modified-GPSR protocols. Results show that there is a significant decrease in the average end-to-end delay of MPR compared with modified-GPSR and BHAG especially at low vehicular density. For instance, at vehicular density of 5 vehicles $/ \mathrm{km}$, the average end-to-end delay of MPR is reduced by $87 \%$ and $83 \%$ compared with modified-GPSR and BHAG, respectively. However, this improvement decreases to $50 \%$ and $2 \%$ at vehicular density of 30 vehicles/ $\mathrm{km}$ due to the increase of the vehicular density that increases the connectivity of the network. In addition, at vehicular density of 15 vehicles $/ \mathrm{km}$, the average endto-end delay of MPR is reduced by $66 \%$ and $50 \%$ compared with modified-GPSR and BHAG, respectively. The reason behind this

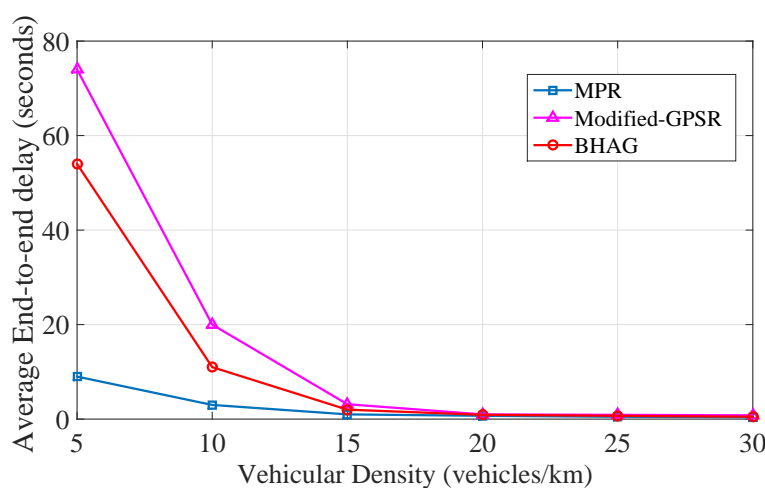

Fig. 1. Average End-to-end Delay.

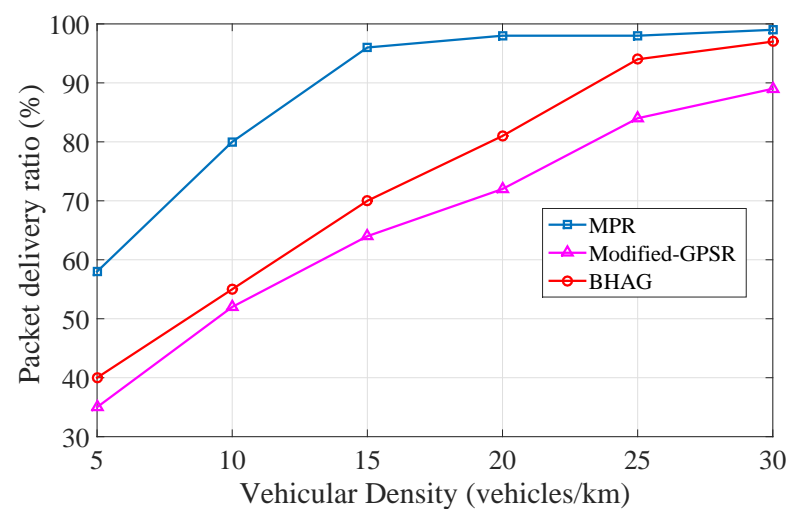

Fig. 2. Packet delivery Ratio.

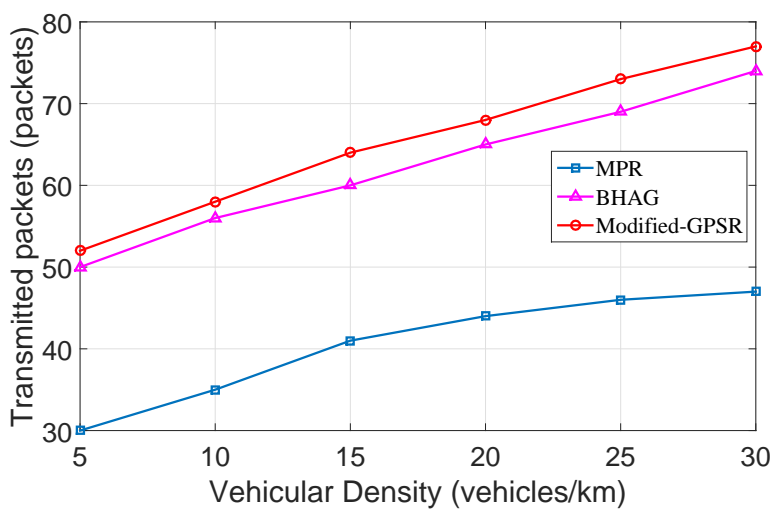

Fig. 3. Routing overhead represented in transmitted packets.

behavior is that low vehicular density leads to disconnected road segment. As a result, the three routing protocols switch to the Carry Mode. Consequently, the packets suffer from a higher end-to-end delay. Also, the results show that the vehicular density highly impacts the end-to-end delay. With decreasing the vehicular density, the average end-to-end delay increases for all values of the vehicular density for the three routing protocol. On the other hand, BHAG has a slightly shorter end-to-end delay than modified-GPSR due to micro topology consideration in the routing metric, especially at 


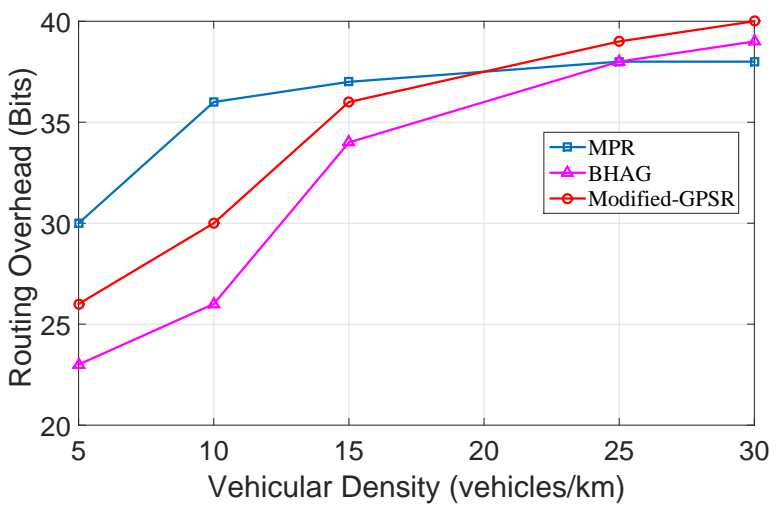

Fig. 4. Routing overhead represented in transmitted bits.

low vehicular density. For instance, at vehicular density of 5 vehicles $/ \mathrm{km}$, the average end-to-end delay of BHAG is reduced by $27 \%$ compared with modified-GPSR.

Fig. 2 shows the PDR against the vehicular density for MPR, BHAG, and modified-GPSR protocols. It is noticed that there is a significant increase in the PDR of MPR compared with modifiedGPSR and BHAG for all values of the vehicular density. For instance, at vehicular density of 5 vehicles $/ \mathrm{km}$, the PDR of MPR is increased by $63 \%$ and $45 \%$ compared with modified-GPSR and BHAG, respectively. However, this improvement decreases to $11 \%$ and $2 \%$ at vehicular density of 30 vehicles $/ \mathrm{km}$ due to the increase of the vehicular density that enhances the connectivity of the network. Three reasons are behind this behavior. Firstly, in BHAG and modified-GPSR, packets are more likely to collide with the beacon packets. On the contrary, MPR is a beacon-less protocol. Secondly, in case of BHAG and modified-GPSR, the single-path of the packet may have in the disconnected road segments. Thirdly, modifiedGPSR depends on the neighbor table to select the next hop. However, the neighbor table may contain outdated information. Consequently, the packet is dropped after forwarding to a non-existing neighbor. On the other hand, MPR does not suffer from the three previous problems as it sends the packet over multiple paths. Therefore, if one copy of the packet is dropped, another copy arrives at the destination. As a result, the PDR of MPR remains the highest of all of them for all values of the vehicular density. BHAG appears to have a slightly higher PDR than modified-GPSR due to micro topology consideration in the routing metric, especially at low vehicular density. For instance, at vehicular density of 5 vehicles $/ \mathrm{km}$, the PDR of BHAG is increased by $14 \%$ compared with that modified-GPSR.

Fig. 3 shows the routing overhead represented in the number of transmitted packets against the vehicular density for MPR, BHAG, and modified-GPSR protocols. It is noticed that MPR has less routing overhead than BHAG and modified-GPSR for all values of the vehicular density. For instance, at vehicular density of 5 vehicles $/ \mathrm{km}$, the routing overhead of MPR is decreased by $40 \%$ and $42 \%$ compared with modified-GPSR and BHAG, respectively. There are two reasons for this behavior. Firstly, MPR is a beaconless routing protocol. Secondly, MPR has the highest number of successfully received data packets compared with BHAG and modified-GPSR. The results confirm that the increase of the vehicular density causes an increase in the routing overhead for all three routing protocols. This is expected because increasing vehicular density leads to an increase in the hop count for the packets.
Moreover, the number of transmitted beacon packets increase in case of BHAG and modified-GPSR with the increase of the vehicular density. BHAG appears to have a marginally higher routing overhead than modified-GPSR due to the beacon packets to collect vehicle information in local micro topology. For instance, at vehicular density of 5 vehicles $/ \mathrm{km}$, the routing overhead of BHAG is increased by $4 \%$ compared with modified-GPSR.

Fig. 4 shows the routing overhead represented in the number of transmitted bits against the vehicular density for MPR, BHAG, and modified-GPSR protocols. It is noticed that MPR has a higher routing overhead than BHAG and modified-GPSR in low vehicular density. For instance, at vehicular density of 5 vehicles $/ \mathrm{km}$, the routing overhead of MPR is increased by $11 \%$ and $30 \%$ compared with modified-GPSR and BHAG, respectively. The reason behind this behavior is that MPR is multi-path routing protocol, while BHAG and modified-GPSR are single-path protocols. In addition, the data packets are larger in size than the beacon packets. On the other hand, the results confirm that MPR overhead remains constant after reaching its peak. However, Modified-GPSR and BHAG overhead increases with the increasing of the vehicular density. For instance, at vehicular density of 30 vehicles $/ \mathrm{km}$, the routing overhead of MPR is decreased by $12 \%$ and $8 \%$ compared with modified-GPSR and BHAG, respectively. This is expected because increasing vehicular density leads to an increase in the number of beacon packets in case of BHAG and modifiedGPSR. On the contrary, MPR is beacon-less routing protocol. Finally, modified-GPSR appears to have a marginally higher routing overhead than BHAG due to the larger size of the beacon packets at modified-GPSR.

\section{CONCLUSION}

In this paper, we proposed multi-path routing protocol that aims to reduce the end-to-end delay and increase the packet delivery ratio. MPR is a beacon-less routing protocol that forwards multiple copies of the packets and eliminates unneeded copies at the intersections. We have investigated the vehicular density impact on the VANET routing protocols performance. Simulation results confirm that the vehicular density highly impacts the routing performance in urban VANET communications. In addition, results show that MPR outperforms BHAG and modified-GPSR in terms of the endto-end delay and packet delivery ratio with a slight increase in the routing overhead. Therefor, MPR can be used for delay-sensitive applications. In the future work, we will consider an adaptive routing protocol that switches between mutli-path and single-path to reduce the routing overhead.

\section{REFERENCES}

[1] Irshad A Abbasi, Babar Nazir, Aftab Abbasi, Sardar M Bilal, and Sajjad A Madani. A traffic flow-oriented routing protocol for vanets. EURASIP J Wirel Commun Netw, 2014(1):1-14, 2014.

[2] Kaiheng Chen, Xulei Cao, Dan Sung, et al. A street-centric routing protocol based on micro topology in vehicular ad hoc networks. IEEE Trans. Veh. Technol, 2015.

[3] Jérôme Härri, Fethi Filali, Christian Bonnet, and Marco Fiore. Vanetmobisim: generating realistic mobility patterns for vanets. In Proc. VANET 06, pages 96-97. ACM, 2006.

[4] Moez Jerbi, Sidi-Mohammed Senouci, Tinku Rasheed, and Yacine Ghamri-Doudane. Towards efficient geographic routing in urban vehicular networks. IEEE Trans. Veh. Technol, 58(9):5048-5059, 2009. 
[5] Brad Karp and Hsiang-Tsung Kung. Gpsr: Greedy perimeter stateless routing for wireless networks. In Proc. ACM MobiCom, pages 243-254. ACM, 2000.

[6] Pratap Kumar Sahu, Eric Hsiao-Kuang Wu, Jagruti Sahoo, and Mario Gerla. Bahg: back-bone-assisted hop greedy routing for vanet's city environments. IEEE Trans. Intell. Transp. Syst, 14(1):199-213, 2013.

[7] Renfei Wang, Mohammed Almulla, Cristiano Rezende, and Azzedine Boukerche. Video streaming over vehicular networks by a multiple path solution with error correction. In Proc. IEEE ICC, pages 580-585. IEEE, 2014.

[8] Nawaporn Wisitpongphan, Fan Bai, Priyantha Mudalige, Varsha Sadekar, and Ozan Tonguz. Routing in sparse vehicular ad hoc wireless networks. IEEE J. Sel. Areas Commun, 25(8):1538-1556, Oct. 2007.

[9] Yong Xiang, Zheng Liu, Ruilin Liu, Weizhen Sun, and Wei Wang. Geosvr: A map-based stateless vanet routing. Ad hoc Netw, 11(7):2125-2135, 2013. 